\title{
Bindung und Psychotherapie. Teil 1
}

\author{
Karin Pinter
}

Bindung ist die Basis für die emotionale Entwicklung. Bindungsmuster bilden sich entlang von interaktiven affektiven Regulationsprozessen, die gleichzeitig mit den Pflegehandlungen zwischen Kind und Bezugsperson ablaufen. Diese Regulationsprozesse fördern in früher Kindheit die Bildung von Erlebensmustern besonders stark; sie begleiten uns fortlaufend; sie sind später auch der Weg, auf dem Veränderungsprozesse möglich werden.

Für die psychotherapeutische Arbeit spielen die Erkenntnisse darüber, wie frühe Bindungserfahrungen das innere Erleben organisieren, in verschiedener Hinsicht eine besonders große Rolle. In diesem Themenheft „Bindung und Psychotherapie, Teil 1“ werden verschiedene Themenfelder und aktuelle Ergebnisse der Bindungsforschung präsentiert.

Im Beitrag von Svenja Taubner und anderen wird eine aktuelle deutsche Studie vorgestellt, in der Bindungsmuster von PsychotherapeutInnen in Ausbildung erhoben werden. In der Diskussion der empirischen Befunde zeigt sich, dass ausgeprägte reflexive Fähigkeiten und die positive Besetzung der Versorgerrolle für die Berufswahl als TherapeutInnen einen Einfluss haben. Im Lichte der Bindungsforschung gewinnen auch die Lehrtherapien im Rahmen der Psychotherapieausbildung als neue überlagernde Erfahrungen insofern eine besondere Bedeutung, als sie die Modifikation von unsicheren in sichere Bindungsmuster fördern können.
Den Zusammenhang von spezifischen psychischen Störungen und Bindungserfahrungen gilt der Beitrag von Melitta Fischer-Kern und andere; sie erörtern den Zusammenhang von Depression und Bindungsmuster anhand von theoretischen Ansätzen (Bindungstheorie, Mentalisierung) und empirischen Studien. Mehrere Vignetten aus den Protokollen der AAI-Befragung von stationären depressiven PatientInnen gewähren einen Einblick in deren Bindungsrepräsentationen.

In meinem Beitrag wende ich einzelne Aspekte der Bindungstheorie auf die psychotherapeutische Behandlung in der Praxis an: dazu zählen etwa Sicherheit fördern, Exploration begleiten und nach Brüchen die Verbindung suchen. An Hand einer Vignette aus der Behandlung eines vermeidend gebundenen Klienten erläutere ich die Herausforderung dieses Bindungsmusters.

In einem Originalbeitrag gibt Hermann Spielhofer einen Überblick über eine kontroversielle Debatte zur Intersubjektivität, wie sie unter dem Einfluss von Bindungstheorie und Säuglingsforschung angeregt wurde.

Im folgenden Heft des psychotherapie forum wird als Teil 2 von „Bindung und Psychotherapie“ diese Themenreihe fortgesetzt.

Interessenkonflikt

Es besteht kein Interessenkonflikt. 